\title{
Research Paper: Relationship Between Gender and Development Status in Children
}

Farrin Soleimani ${ }^{1},{ }^{*}$ Zahra Bajalan ${ }^{2}$, Hamid Alavi Majd ${ }^{3}$, Somayeh Fallah ${ }^{2}$

1. Pediatric Neurorehabilitation Research Center, University of Social Welfare and Rehabilitation Sciences, Tehran, Iran.

2. Department of Midwifery, Faculty of Nursing and Midwifery, Qazvin University of Medical Sciences, Qazvin, Iran.

3. Department of Biostatistics, Faculty of Paramedical, Shahid Beheshti University of Medical Sciences, Tehran, Iran.

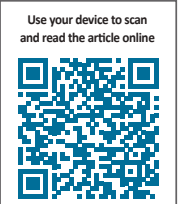

ditation: Soleimani F, Bajalan Z, Alavi Majd H, Fallah S. [Relationship Between Gender and Development Status in Children (Persian)]. Archives of Rehabilitation. 2018; 18(4):338-345. https://doi.org/10.21859/JREHAB.18.4.8

doi)" https://doi.org/10.21859/JREHAB.18.4.8

Received: 03 Jul. 2017 Accepted: 27 Sep. 2017

Keywords:

Gender, Developmental problems, Children, Ages and Stages Questionnaire

\section{A B STR ACT}

Objective Developmental and behavioral problems are the most prevalent problem in pediatrics after infection and trauma. These problems can be affected by numerous factors. A range of factors is involved in behavior and development problems. Developmental problems have a massive impact on children's health and eventually have broader scale effect on society. Because of the necessity for more and different follow-ups in children with developmental disorders in the early years of lifetime, determination of effective factors is essential in any society. Therefore, the current study was conducted with a purpose to determine the relationship between gender and developmental status in children.

Materials \& Methods This is a descriptive design study, performed in 12-month-old children ( $n=250)$ who were referred to health services centers affiliated to Qazvin University of Medical Sciences in 2012. Sampling was performed using a multistage method. The data gathering tool included information form and Age and Stages Questionnaire for measuring developmental status. Once parental consent was obtained, the developmental status of the children was measured in five areas, i.e., motor (gross and fine motor skills), problem-solving, personal-social skills and communication. Age and Stages Questionnaire consists of 19 questionnaires at the age of 4-60 -month old with the aim of separating inactive children from further developmental studies of healthy children. Completion of the questionnaire takes 10 to 15 minutes, and it takes about one minute to score. The validity and reliability of the Age and Stages Questionnaire have been reported in various previous studies. The data were analyzed using SPSS software version 18. In this study, statistical analyses were performed using descriptive and inferential statistics. Logistic regression model was used to determine the relationship between gender and developmental status. A P value of $<0.05$ was considered statistically significant. Results The prevalence of developmental delay was $22.4 \%$ (CI 95\%: 17-28) in children and the highest prevalence was in the area of communication (8\%; $\mathrm{Cl} 95 \%$ : 5-11) and the lowest was in the area of problem-solving (2\%; Cl 95\%; 0-4). 39 boys (69.6\%) were in the group of developmental delay, and 102 boys (52.6\%) were in normal developmental group. To check the status of development difference between groups were used chisquare test showed a significant difference between the two groups. Therefore, developmental delay is significantly higher in boys $(P=0.02)$. By importing the variable studied in the logistic regression model, the male gender variable $(\mathrm{P}=0.055, \mathrm{OR}=0.52)$ had a significant relationship with a developmental delay of children. Children with suspected developmental disorders in evolution children with normal development, chance of developmental delay in boys 0.52 times more than girls. The other variables (age, education, and occupation of parents, previous history of abortion, type of delivery, parity, spacing between births, weight and head circumference at birth and 12 months of age) did not show a significant relationship with developmental delay. Conclusion The leading causes of developmental disabilities remain unknown. It seems that gender is one of the causes of developmental delay. In this study, male gender was a factor that is associated with developmental delay. According to the incidence of developmental delay and its effect on society, it is essential that health professionals, with family, and community, monitor the development of children. Identifying the children at risk as soon as possible for diagnosis, earlier intervention, and reduced problems is essential in the future. Further studies are required to confirm the results.

\section{* Corresponding Author:}

Zahra Bajalan, MSc.

Address: Department of Midwifery, Faculty of Nursing and Midwifery, Qazvin University of Medical Sciences, Qazvin, Iran.

Tel: +98 (283) 3336001

E-Mail: z.bajalan64@gmail.com 


\title{
بررسى ارتباط جنسيت با وضعيت تكاملى كودكان
}

\author{
فرين سليمانى'، "زهرا باجلان'، حميد علوى مجد"، سميه فلاح'

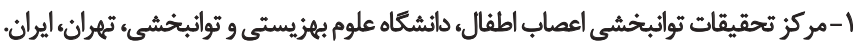

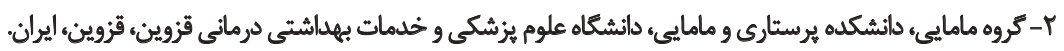

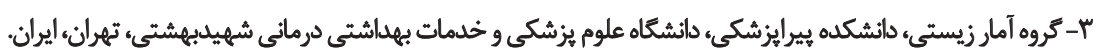

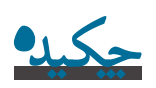

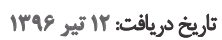

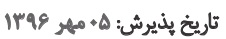

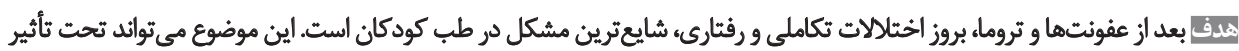

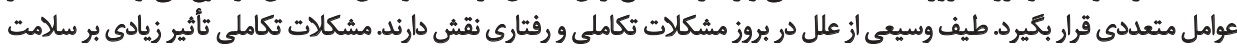

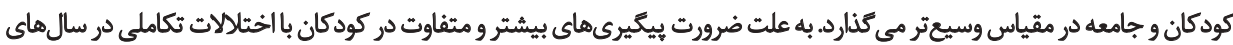

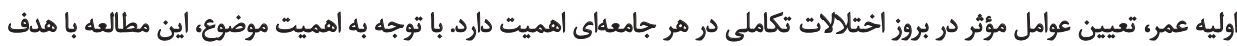

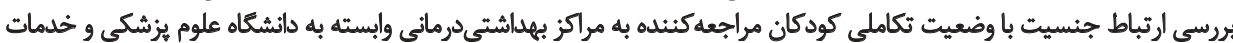

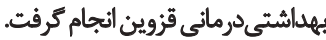

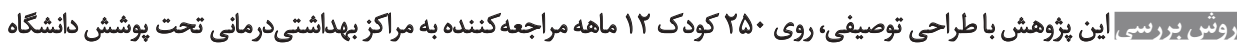

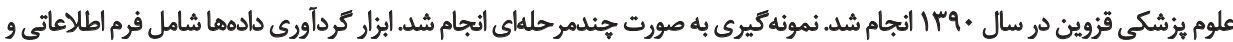

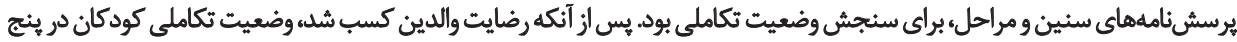

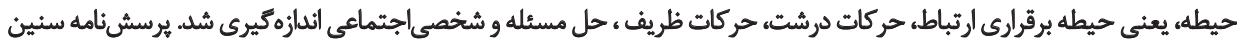

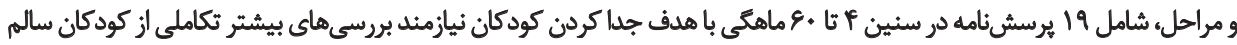

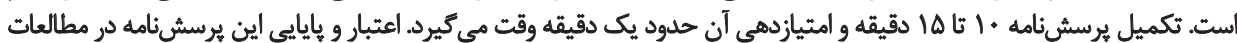

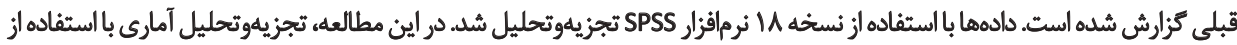

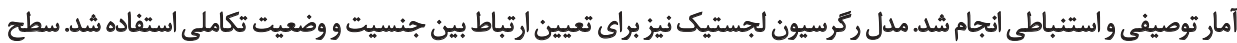

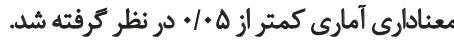

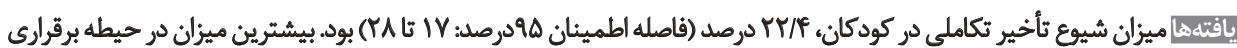

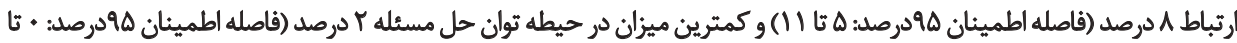

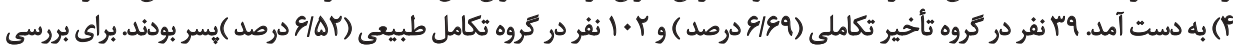

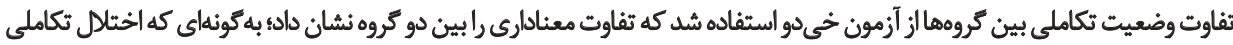

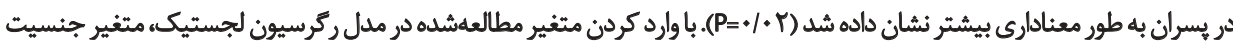

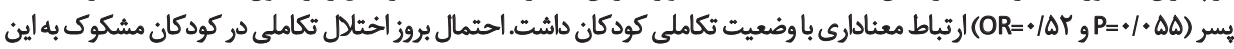

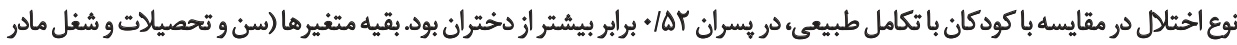

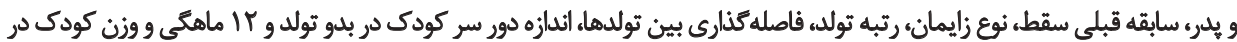

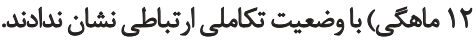

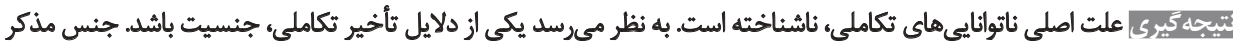

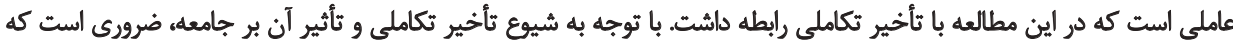

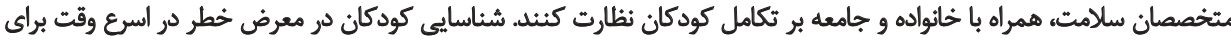

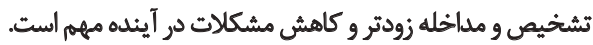




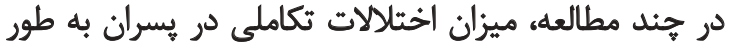

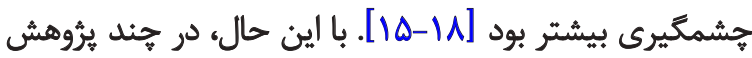

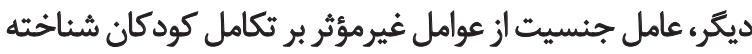

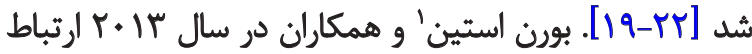

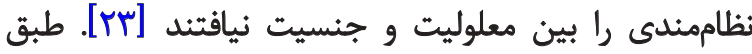

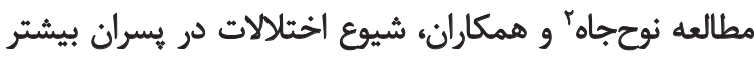

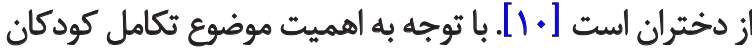

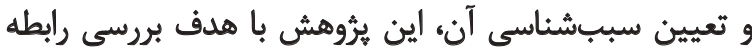

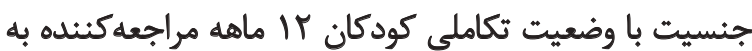

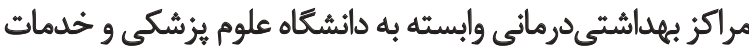

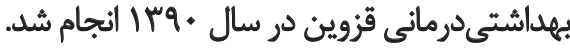

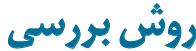

جروهش حاضر، مطالعهاي توصيفى است و در بر آن، ارتباط

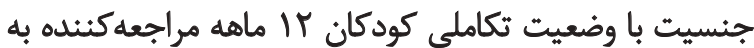

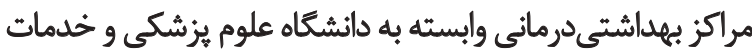

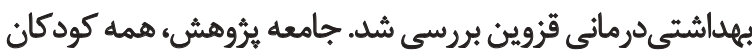

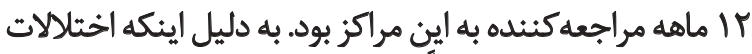

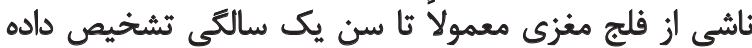

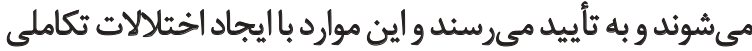
باعث مخدوش شدن نتايج مطالعه مىشوند مورديا

در اين مطالعه معيارهاى ورود كودكان به بثروهش به اين شرح

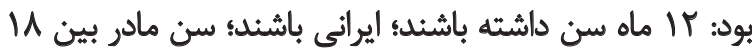

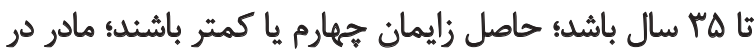

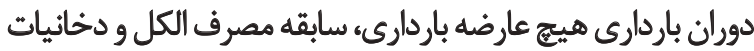

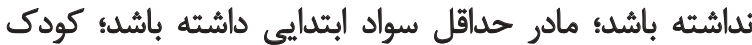

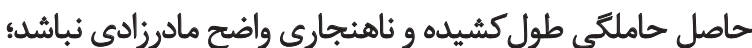

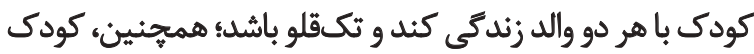

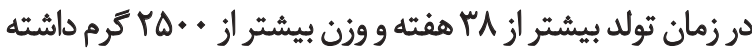

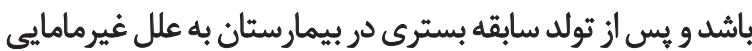

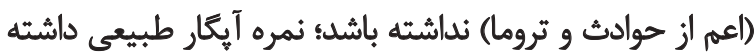

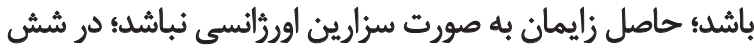

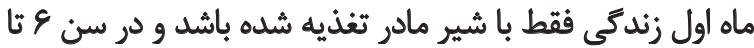

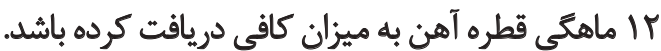

نمونهكيرى به صورت جندمرحلهائ انجام شد. مراكز

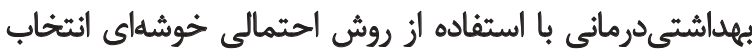

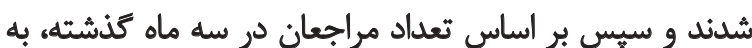

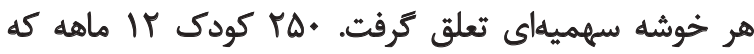

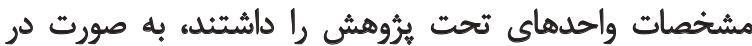

\section{Bornstein}

2. Nouhjah

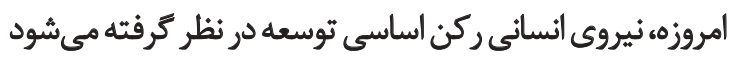

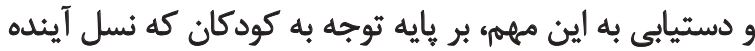

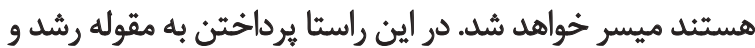

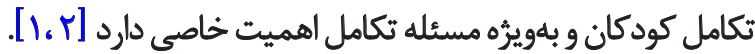

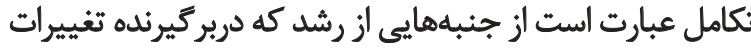

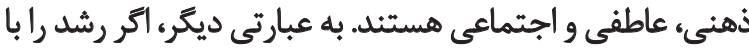

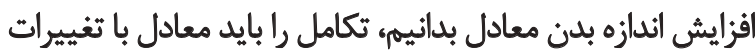
در عمل به حساب آوريم [ب].

هنتامى كه كودك، در كسب توانايىهاى تكاملى مطابق با باني

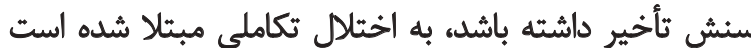

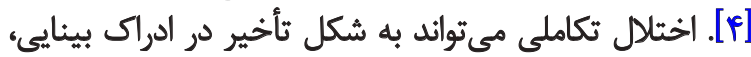

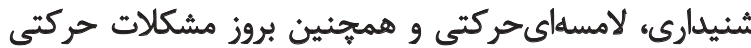

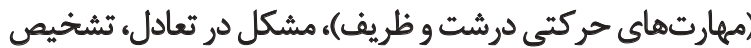

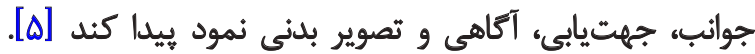

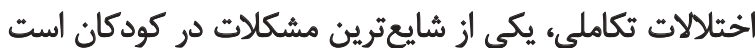

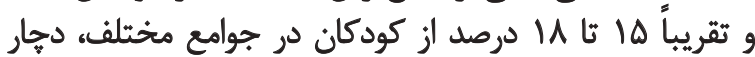

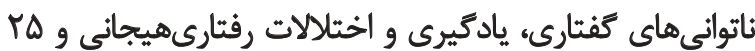

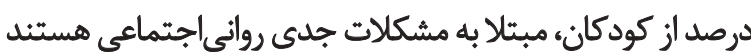

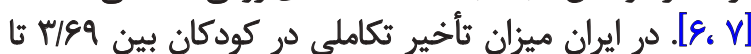

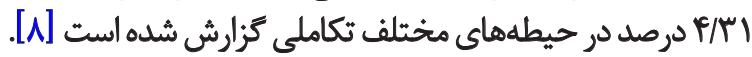

رشد و تكامل، از كودكى به كودك ديكر متغير است و اين تونين

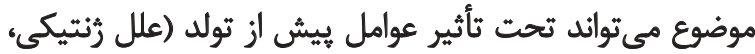

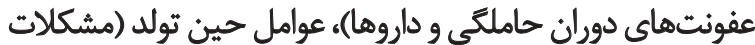

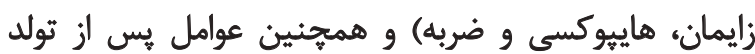

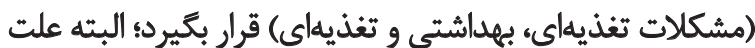

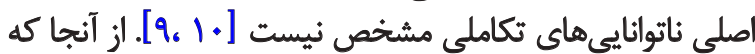

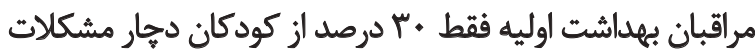

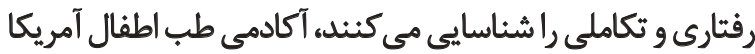

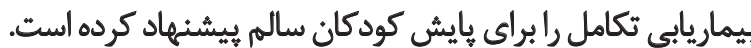

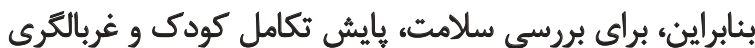

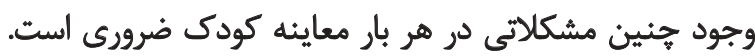

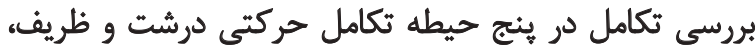
شناختى، عاطفى ارثباطى (دركى و بيانى ) و حلى حل مسئله صورت مي كيرد [11]. مطالعات انجامشده درباره رابطه جنسيت باوضعيت تكاملى نتايج

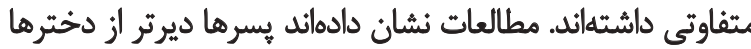

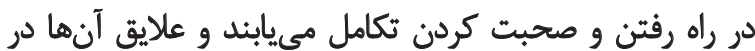

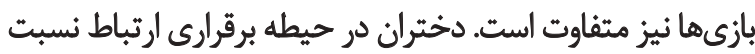

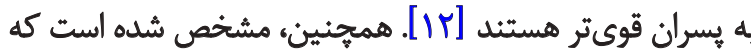

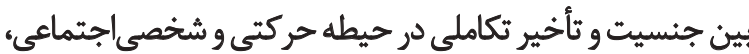

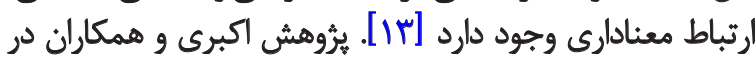


آزمون مجدد يك هفته بعد، با همان · إنفر سنجيله شد. سيس،

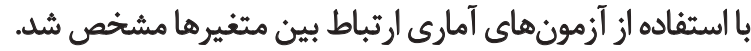
يرسشنامه آزمون تكاملي سنين و مراحل، ابزارى معتبر در

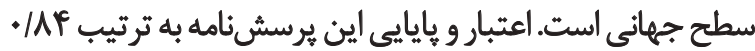

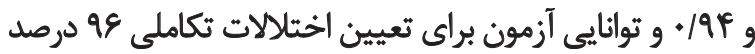

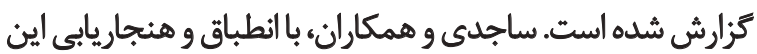

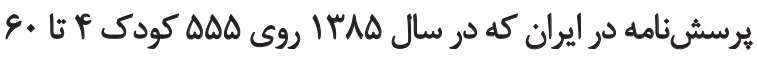

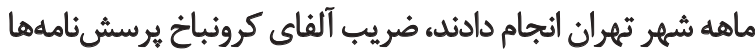

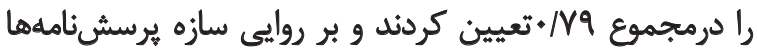

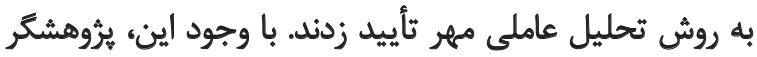

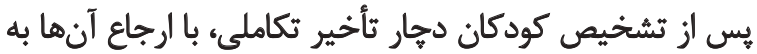

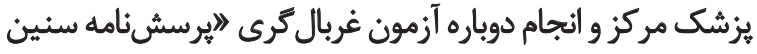

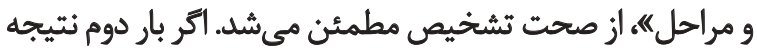

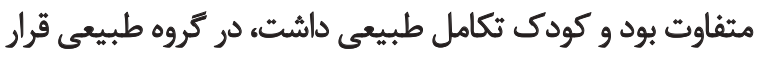

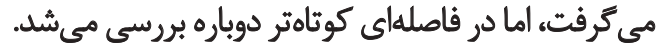
يس از ثأييد كميته اخلاق و ارائه معرفيى نامه رسمى به مسئولان

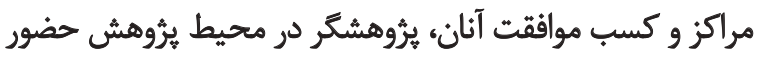

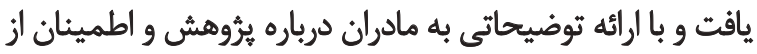

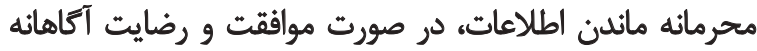

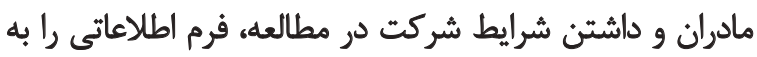

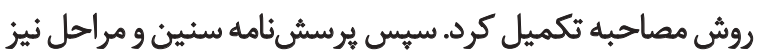

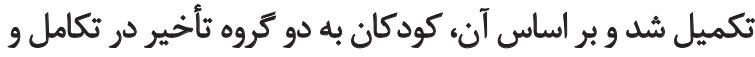

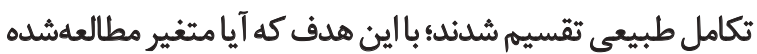

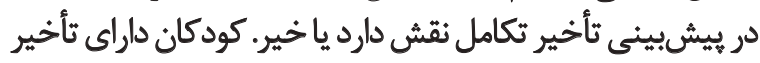

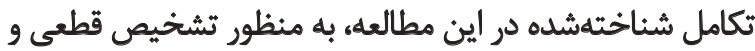

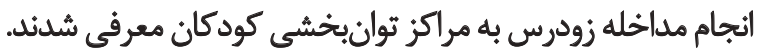

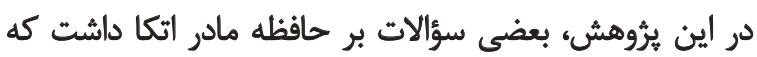

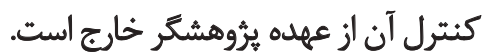

براى تجزيلوتحليل دادهها از نسخه ل1 انرمافزار SPSS استفاده

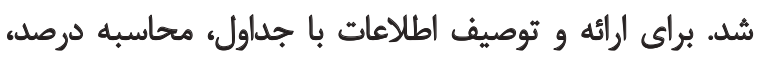

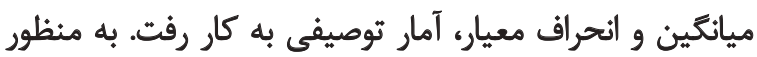

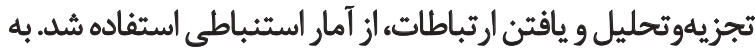

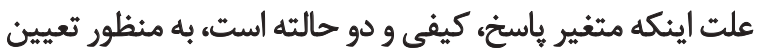

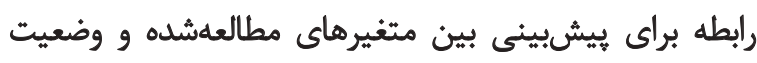

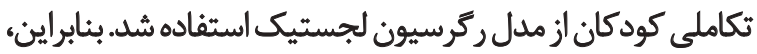

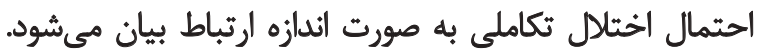

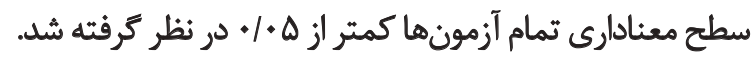

Ldió

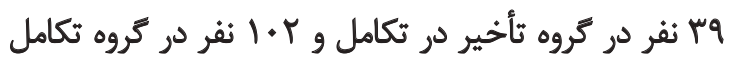

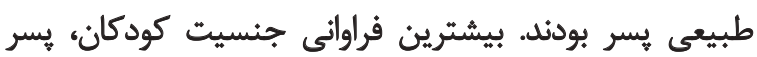

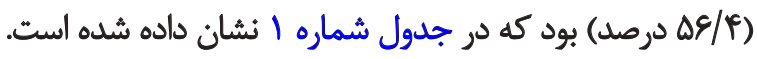

دسترس نمونهكيرى شديند و والدين يرسشنامه سنين و مراحل

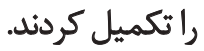

ابتدا فهرست همه مراكز بهداشتىدرمانى وابسته به دانشكاه علوم

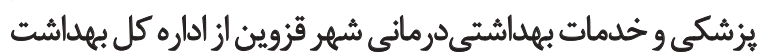

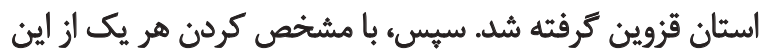

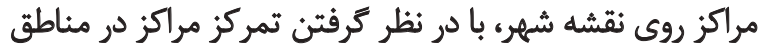

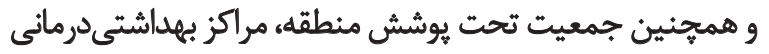

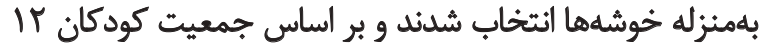

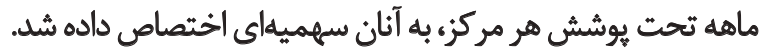

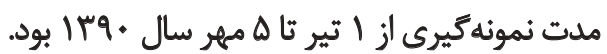

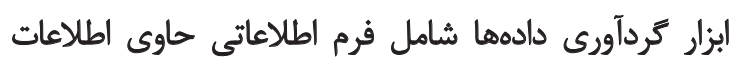

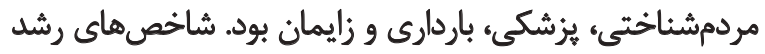

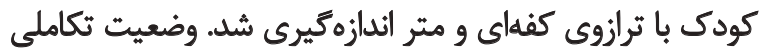

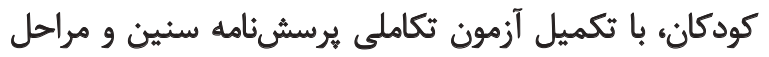

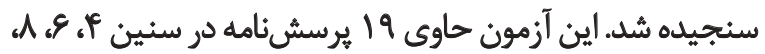

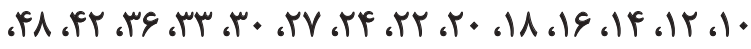

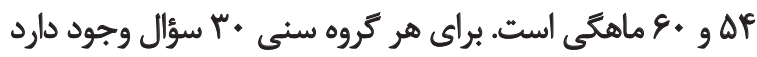

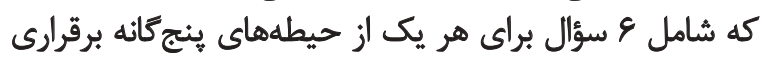

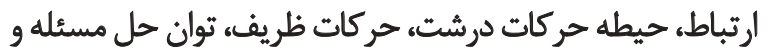

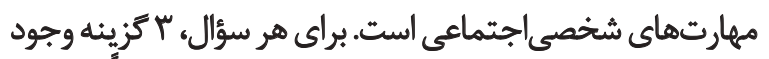

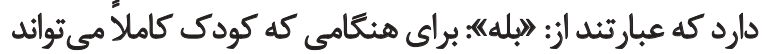

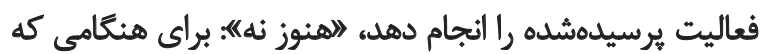

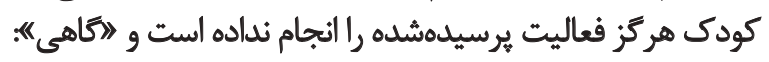

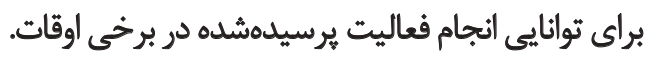

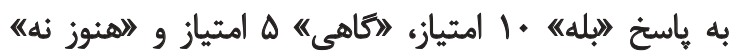

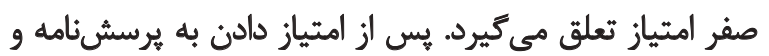

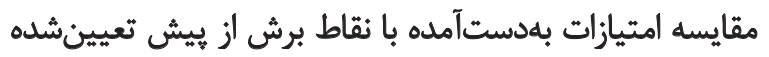

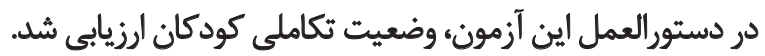

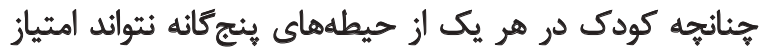

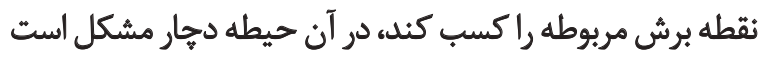

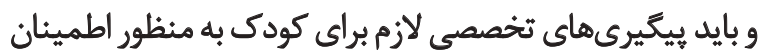

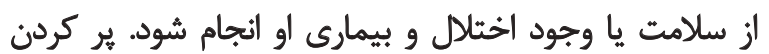

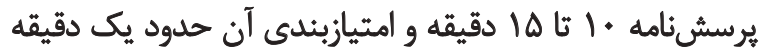
وقت مي كيرد [1f]

در خصوص اعتباريابى فرم اطلاعاتى از روش اعتبار فرم

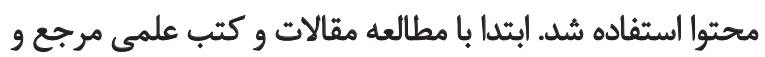

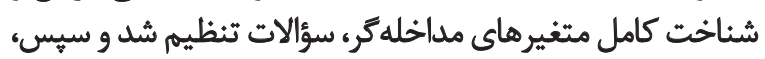

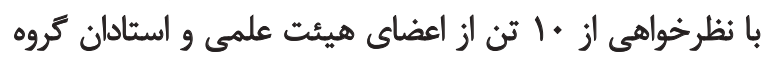

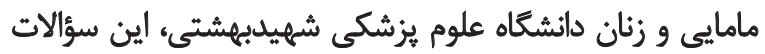

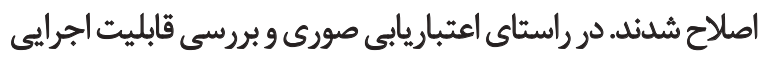

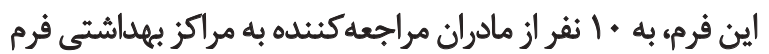

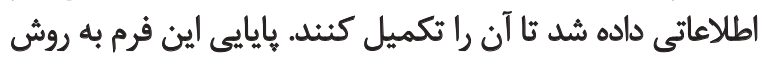


جدول ا. فراوائى اختّلال تكامل و تكامل طبيعى در كودكان

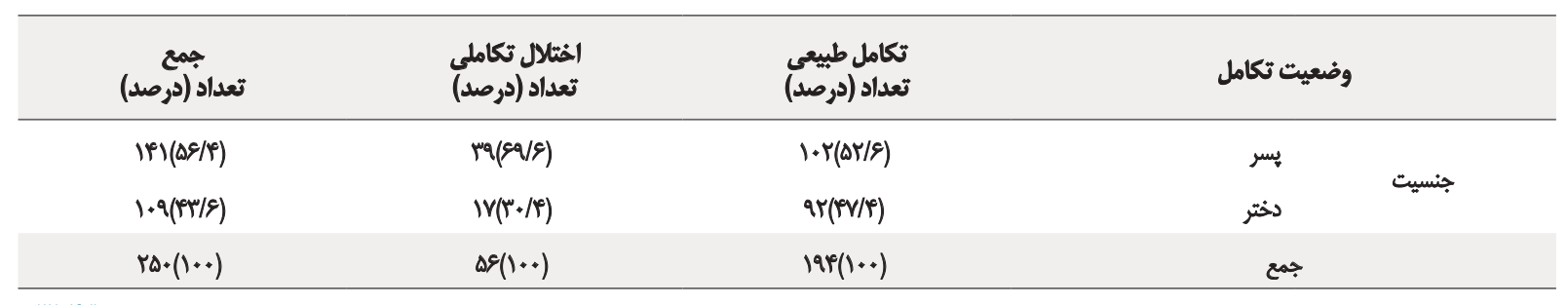

توانبخننى

جدول ץ. ارتباط متغير سن والدين و جنسيث با وضعيت ثكاملي كودكان

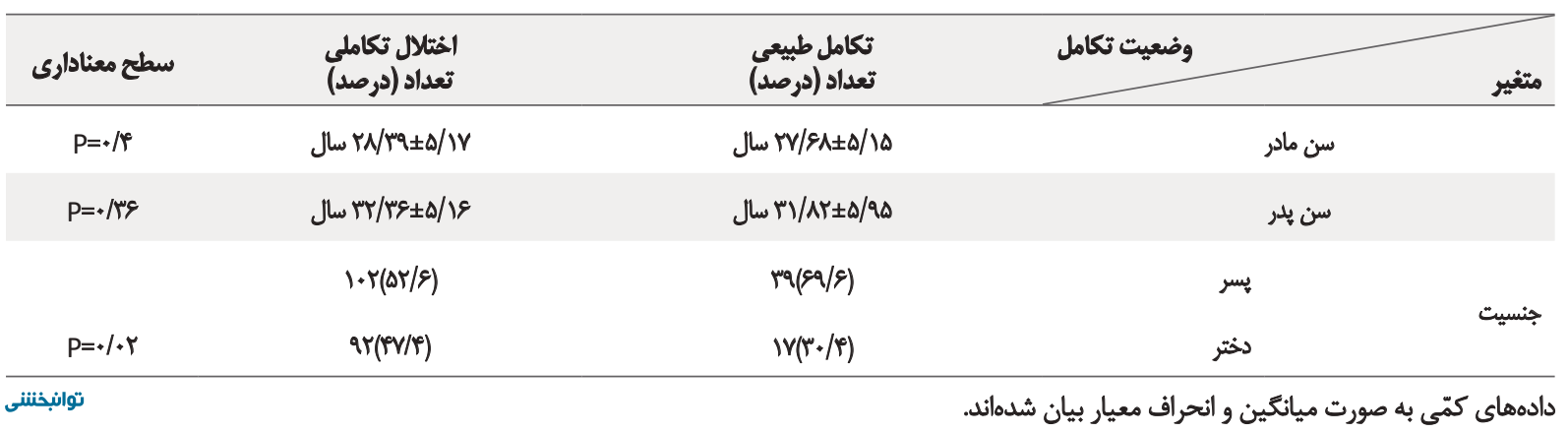

يدر، سابقه قبلى سقط، نوع زايمان، رتبه تولد، ازدواج فاميلى،

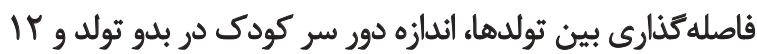

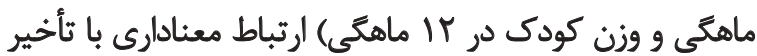
تكاملى نداشتند (ه> (P> (P) ).

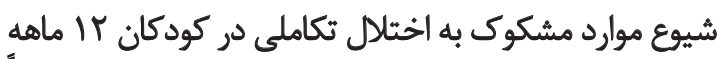

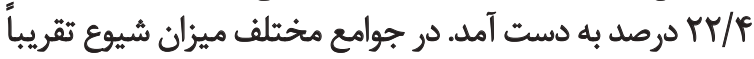

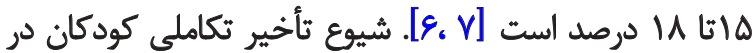

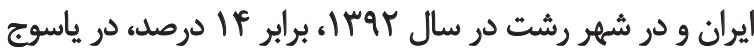

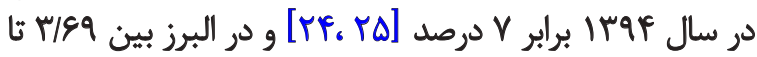

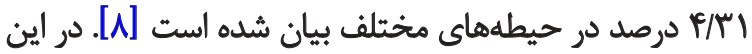

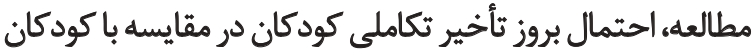

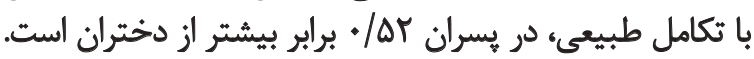

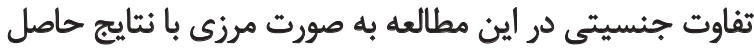

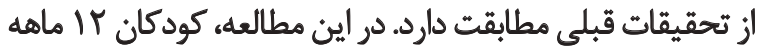

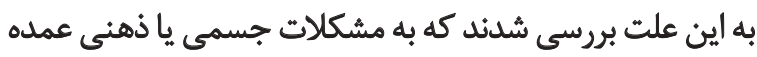

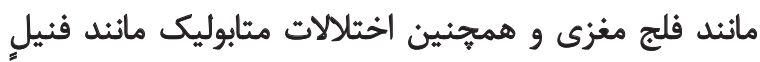

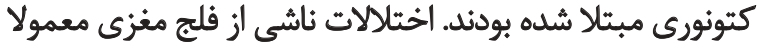

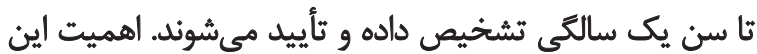

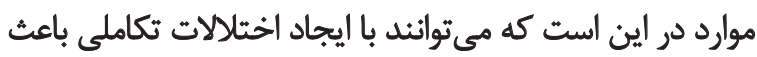
مخدوش شدن نتايج مطالعه شوند.

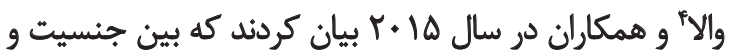

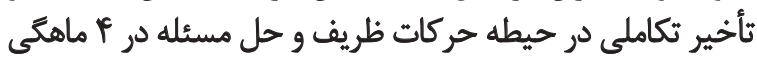

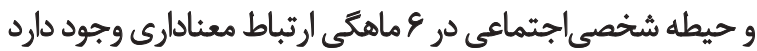

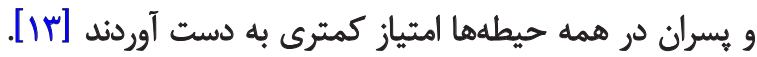

شيوع موارد مشكوك به اختلال تكاملى در كودكان در مطالعه

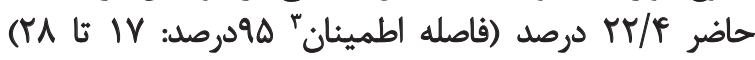
بود. بيشترين ميزان در حيطه برقرارى ارتباط \ 1 درصد (فاصله

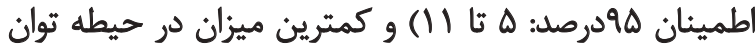

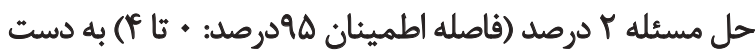

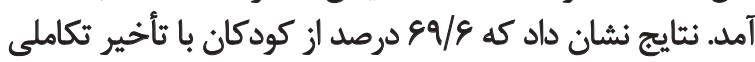

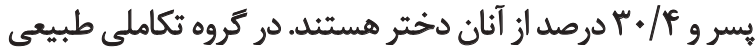

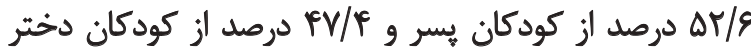

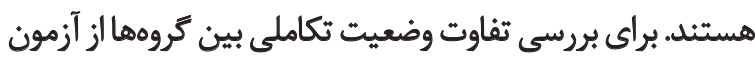

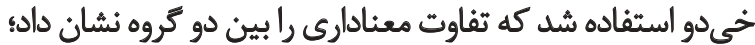

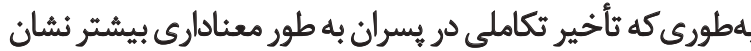

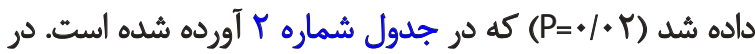

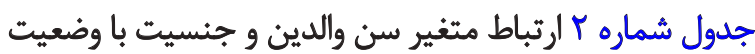
تكاملى كودكان بيان شده است.

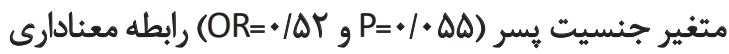

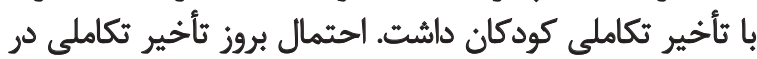

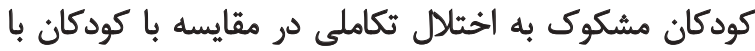

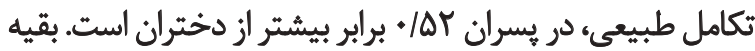
متغيرها رابطه معنادارى با تأخير تكاملى نشان بران ندادئد.

\section{ث0}

نتيجه اين يُورهش نشان داد جنسيت يسر در مدل لجستيك

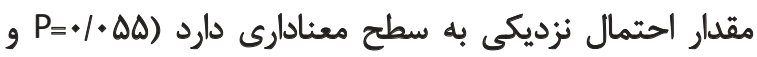
(OR=•/Dr 
شايد عامل جنسيت، رابطه معنادارى با اختلال تكاملى در

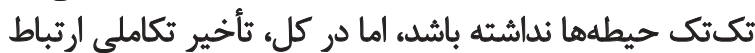

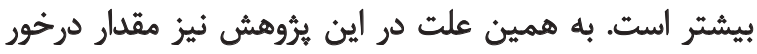

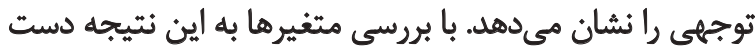

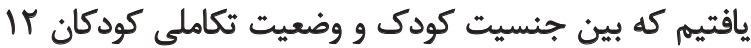

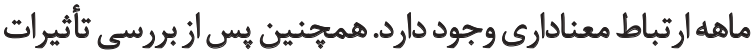

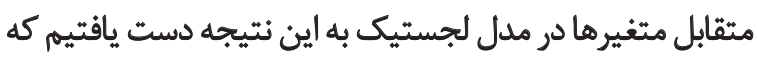

يسر بودن ارتباط معنادارى با وضعيت ثكاملى كودكان دارد.

\section{نتيجهيَّيرى}

با توجه به شيوع تأخير تكاملى و ثأثير آن بر جامعه، ضرورى

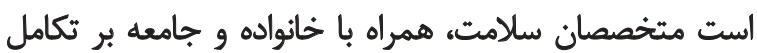

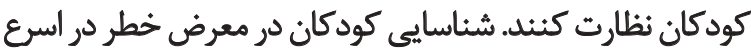

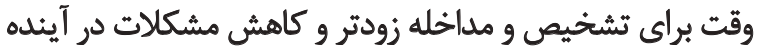

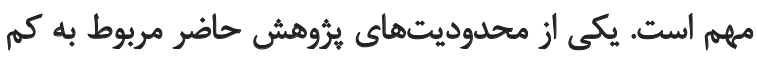

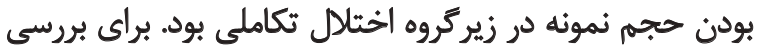

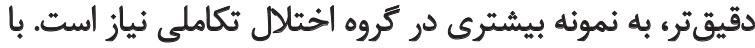

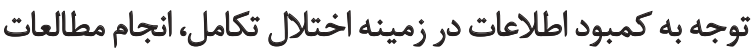
مشابه در اين زمينه ضرورى استلات در زمينه

$$
\text { تشكر وقمدرانى }
$$

اين مقاله حاصل بايانانامه مقطع كارشناسى ارشد خانم زهرا

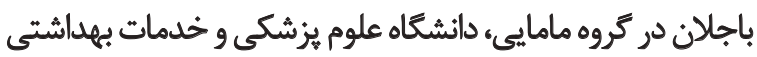

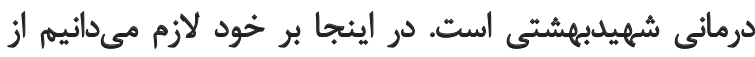

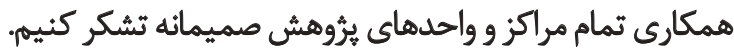

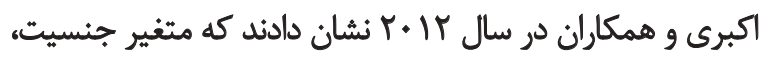

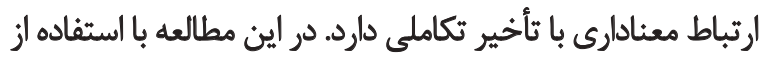

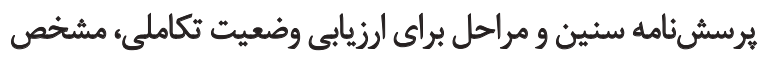

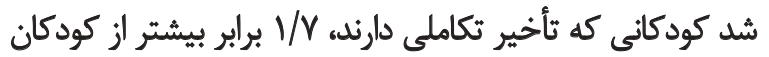

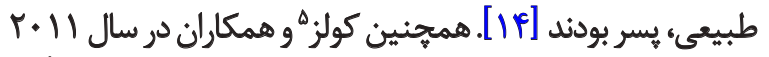
بيان كردند كه در ميان كودكان جنسيت بر بر رشد حركني

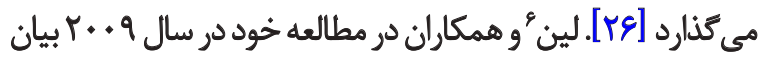

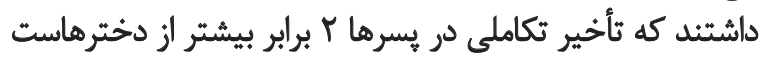

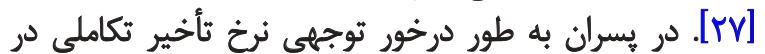

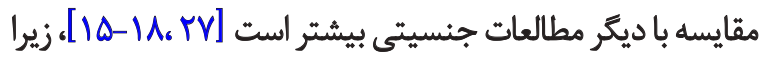

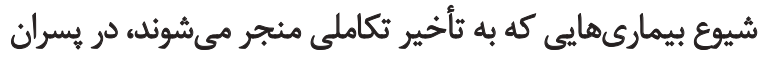

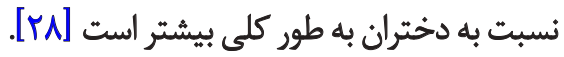

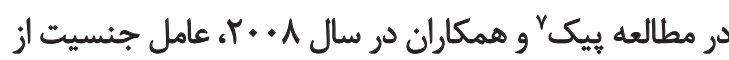

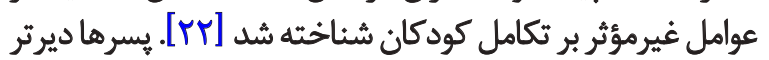

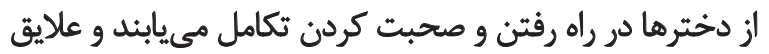

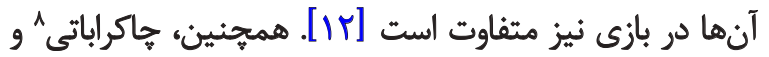

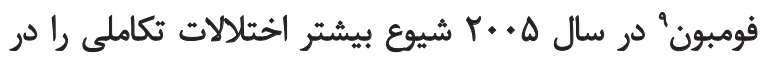

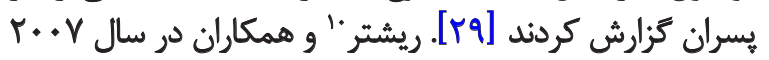

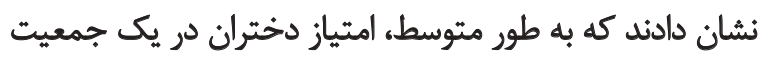

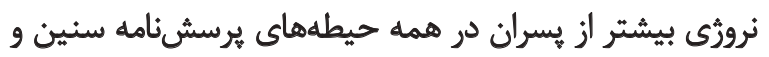

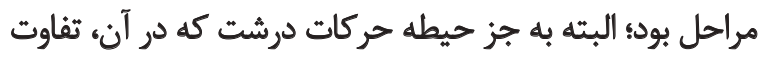

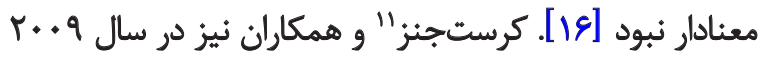

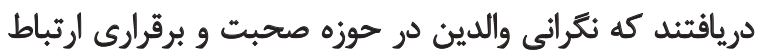

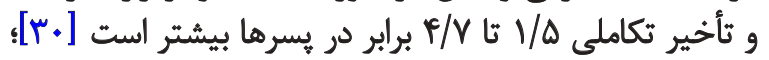

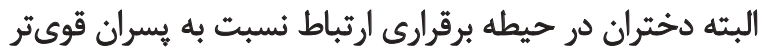

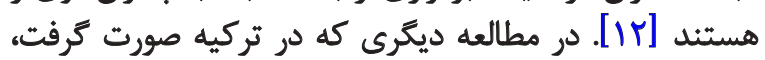

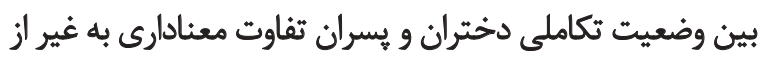

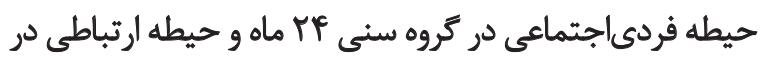

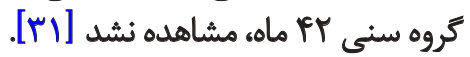

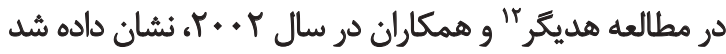

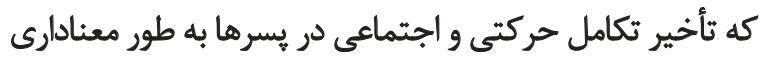

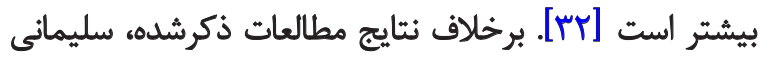

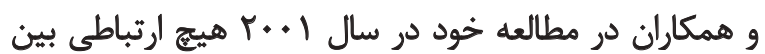

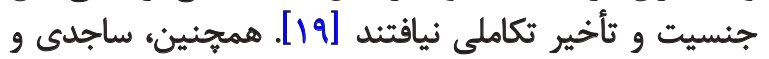

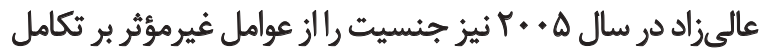

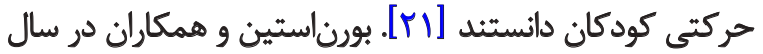

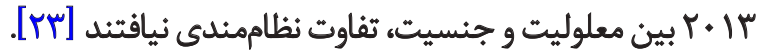

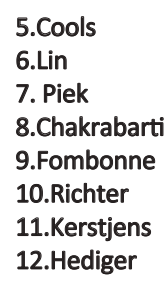




\section{References}

[1] Kosaryan M, Vahidshahi K, Shafaat B, Abaskhanian A, Azizi S, Shahrokh SH, Asadi M. [Screening of developmental problem, day care centers, Sari, 2006 (Persian)]. Journal of Mazandaran University of Medical Sciences. 2007; 17(59):69-75.

[2] Soleimani F, Sajedi F, Akbari SA. Developmental delay and related factors (Persian)]. Advances in Nursing \& Midwifery. 2014; 24(85):61-70.

[3] Mohammadi Parsa N, Moradi A, Einabadi F. [An investigation of the relationship between maternal depression with gross motor and fine motor delay in one -year old children (Persian)]. Journal of Paramedical and Rehabilitation Sciences. 2015; 4(4):43-50.

[4] Karimzadeh P. [Development and childhood developmental problems (Persian)]. Tehran: University of Social Welfare and Rehabilitation Sciences; 2005

[5] Siminghalam M, Alibakhshi H, Ahmadi zadeh Z. [An investigation of bilateral coordination of children with specific learning disorder (Persian)]. Journal of Paramedical and Rehabilitation Sciences. 2016; 5(1):7-13.

[6] Glascoe FP. Early detection of developmental and behavioral problems. Pediatrics in Review. 2000; 21(8):272-80. doi: 10.1542/ pir.21-8-272

[7] Rydz D, Srour M, Oskoui M, Marget N, Shiller M, Birnbaum $\mathrm{R}$, et al. Screening for developmental delay in the setting of a community pediatric clinic: A prospective assessment of parentreport questionnaires. Pediatrics. 2006; 118(4):e1178-e1186. doi: 10.1542/peds.2006-0466

[8] Sajedi F, Vameghi R, Kraskian Mujembari A. Prevalence of undetected developmental delays in Iranian children. Child: Care, Health and Development. 2013; 40(3):379-88. doi: 10.1111/ cch.12042

[9] Fox JA. Primary health care of children. New York: Mosby; 1997.

[10] Nouhjah S, Mokhveli Khazaei F. [Assessment of motor development of children attending health centers of Dezful City using World Health Organization standard indexes (Persian)]. Journal of Paramedical Sciences \& Rehabilitation. 2014; 3(1):16-26.

[11] Behrman RE, Jenson HB, Stanton BF. Nelson textbook of pediatrics. Philadelphia: Saunders; 2007.

[12] McGregor KK, Oleson J, Bahnsen A, Duff D. Children with developmental language impairment have vocabulary deficits characterized by limited breadth and depth. International Journal of Language \& Communication Disorders. 2013; 48(3):307-19. doi: $10.1111 / 1460-6984.12008$

[13] Valla L, Wentzel Larsen T, Hofoss D, Slinning K. Prevalence of suspected developmental delays in early infancy: Results from a regional population-based longitudinal study. BMC Pediatrics. 2015; 15(1). doi: 10.1186/s12887-015-0528-z

[14] Amir-Ali-Akbari S, Torabi F, Soleimani F, Alavi-Majd H. [Correlation between high risk pregnancy and developmental delay in children 4-60 months in Isfahan, Iran 2010-2011 (Persian)]. Archives of Rehabilitation. 2011; 11:40-49

[15] Berglund E, Eriksson M, Westerlund M. Communicative skills in relation to gender, birth order, childcare and socioeconomic status in 18-month-old children. Scandinavian Journal of Psychology. 2005; 46(6):485-91. doi: 10.1111/j.1467-9450.2005.00480.x

[16] Richter J, Janson H. A validation study of the Norwegian version of the Ages and Stages Questionnaires. Acta Paediatrica. 2007; 96(5):748-52. doi: 10.1111/j.1651-2227.2007.00246.x

[17] Rydell AM, Diamantopoulou S, Thorell LB, Bohlin G. Hyperactivity, shyness, and sex: Development and socio-emotional functioning. British Journal of Developmental Psychology. 2009, 27(3):625-48. doi: 10.1348/026151008x346996

[18] Simpson GA, Colpe L, Greenspan S. Measuring functional developmental delay in infants and young children: Prevalence rates from the NHIS-D. Paediatric and Perinatal Epidemiology. 2003; 17(1):68-80. doi: 10.1046/j.1365-3016.2003.00459.x

[19] Soleimani F, Khoshbin E, Shams S. [Report of motor developmental delay screening of infants (4-18 Months Old) of Karaj city (Persian)]. Archives of Rehabilitation. 2001; 2(3):22-8.

[20] Sajedi F, Vameghi R, Habibollahi A, Lornejad H, Delavar B. Standardization and validation of the ASQ developmental disorders screening tool in children of Tehran city. Tehran University of Medical Sciences. 2012; 70(7):436-46.

[21] Sajedi F, Ali Zadeh V. [Study of prevalence and influence fac tor in delayed motor development in high risk infants (Persian)]. Archives of Rehabilitation. 2005; 5(4):7-12.

[22] Piek JP, Dawson L, Smith LM, Gasson N. The role of early fine and gross motor development on later motor and cognitive ability. Human Movement Science. 2008; 27(5):668-81. doi: 10.1016/j.humov.2007.11.002

[23] Bornstein MH, Hendricks C. Screening for developmental disabilities in developing countries. Social Science \& Medicine. 2013; 97:307-15. doi: 10.1016/j.socscimed.2012.09.049

[24] Khabbazkar F, Dolatian M, Soleimani F, Alavi Majd H. [The survey of the correlation between domestic violence during pregnancy and after delivery with developmental status of 8-12 month's infant (Persian)]. Pejouhandeh. 2015; 19(6):320-7.

[25] SF A, Mahboobe A, Firoozeh S. [Development status of 4-24 months children born to teenage mothers referred to health care centers in Yasuj, 2013 (Persian)]. Armaghan-e Danesh. 2015; 20(3):253-63

[26] Cools W, De Martelaer K, Samaey C, Andries C. Fundamental movement skill performance of preschool children in relation to family context. Journal of Sports Sciences. 2011; 29(7):649-60. doi: $10.1080 / 02640414.2010 .551540$

[27] Lin JD, Yen CF, Wu JL, Kang SW. The administrative population report on children with developmental delays in Taiwan, 2003 through 2007. Research in Developmental Disabilities. 2009; 30(2):353-8. doi: 10.1016/j.ridd.2008.06.004

[28] Lai DC, Tseng YC, Guo HR. Gender and geographic differences in developmental delays among young children: Analysis of the data from the national registry in Taiwan. Research in Developmental Disabilities. 2011; 32(1):63-9. doi: 10.1016/j. ridd.2010.08.012

[29] Chakrabarti S, Fombonne E. Pervasive developmental disorder in preschool children: Confirmation of high prevalence. Ameri- 
can Journal of Psychiatry. 2005; 162(6):1133-41. doi: 10.1176/ appi.ajp.162.6.1133

[30] Kerstjens JM, Bos AF, ten Vergert EMJ, de Meer G, Butcher PR, Reijneveld SA. Support for the global feasibility of the Ages and Stages Questionnaire as developmental screener. Early Human Development. 2009; 85(7):443-7. doi: 10.1016/j.earlhumdev.2009.03.001

[31] Kapci EG, Kucuker S, Uslu RI. How applicable are ages and stages questionnaires for use with Turkish children. Topics in Early Childhood Special Education. 2010; 30(3):176-88. doi: 10.1177/0271121410373149

[32] Hediger ML, Overpeck MD, Ruan WJ, Troendle JF. Birthweight and gestational age effects on motor and social development. Paediatric and Perinatal Epidemiology. 2002; 16(1):33-46. doi: 10.1046/j.1365-3016.2002.00393.x 